\title{
Backward running or absence of running from Creutz ratios
}

\author{
Joel Giedt** \\ Department of Physics, Applied Physics and Astronomy, Rensselaer Polytechnic Institute, Troy, \\ New York \\ E-mail: giedtjerpi.edu
}

\section{Evan Weinberg}

E-mail: weinbe2@rpi.edu

\begin{abstract}
We extract the running coupling based on Creutz ratios in SU(2) lattice gauge theory with two Dirac fermions in the adjoint representation. Depending on how the extrapolation to zero fermion mass is performed, either backward running or an absence of running is observed at strong bare coupling. This behavior is consistent with other findings which indicate that this theory has an infrared fixed point.
\end{abstract}

The XXIX International Symposium on Lattice Field Theory - Lattice 2011

July 10-16, 2011

Squaw Valley, Lake Tahoe, California

\footnotetext{
${ }^{*}$ Speaker.

${ }^{\dagger}$ Supported by the Department of Energy, Office of Science, Office of High Energy Physics
} 


\section{Motivation}

Technicolor $[1,2]$ was quickly specialized to walking technicolor in order to address flavorchanging neutral currents $[3,4,5,6,7,8,9]$. Higher representations may avoid problems with the S-parameter [10, 11]. This motivates the study of Minimal Walking Technicolor [12] using lattice techniques, in order to see where this theory lies with respect to the conformal window. Does it really walk? Here Creutz ratios suggest and answer. The approach is different from the Schrödinger functional method that was employed for SU(3) gauge group with triplet fermions in [13], SU(3) gauge group with sextet fermions [14], and Minimal Walking Technicolor [15, 16, 17]. The latter works indicate the existence of an infrared fixed point. We obtain results consistent with that conclusion by this alternate Creutz ratio approach.

\section{The method}

In [18] a method for measuring the running gauge coupling on the lattice using Creutz ratios was proposed. In [19, 20] this method was applied to pure SU(3) lattice Yang-Mills, and in [20, 21] this method was applied in a preliminary way to $\mathrm{SU}(3)$ gauge group with sixteen triplet fermions, and more recently in [22] to SU(2) gauge group with eight flavors of fundamental representation fermions. We will use this approach to search for the phenomenon of backward running, which is a smoking gun for a nontrivial infrared fixed point, since forward running is assured at weak coupling by perturbative methods. We apply the method of Creutz ratios to the theory of Minimal Walking Technicolor: $\mathrm{SU}(2)$ gauge theory with two flavors of fermions in the adjoint representation. Our lattice action is Wilson fermions and a plaquette gauge action.

\subsection{Outline}

The proposal [18] defines the running coupling $g(L)$ at a scale $L=N a$ associated with the spatial extent of the lattice, where $N$ is the number of sites in spatial directions and $a$ is the lattice spacing. ${ }^{1}$ This is done through the Creutz ratio [23]:

$$
\chi(I, J)=-\ln \frac{W(I, J) W(I-1, J-1)}{W(I, J-1) W(I-1, J)}
$$

where $W(I, J)$ is the expectation value of the trace of the rectangular $I \times J$ Wilson loop on the lattice. Throughout, we only consider $\chi(I, I)$. The Creutz ratio is interpolated between values of $I$ to define a related function $\tilde{\chi}(\hat{R})$ with $\hat{R} \equiv R / a$ continuous. The interpolation requires a matching of values at the points where $\hat{R}$ is half-integral:

$$
\tilde{\chi}(\hat{R}) \equiv \chi\left(\hat{R}+\frac{1}{2}, \hat{R}+\frac{1}{2}\right)=-\ln \frac{W\left(\hat{R}+\frac{1}{2}, \hat{R}+\frac{1}{2}\right) W\left(\hat{R}-\frac{1}{2}, \hat{R}-\frac{1}{2}\right)}{W\left(\hat{R}+\frac{1}{2}, \hat{R}-\frac{1}{2}\right)^{2}}
$$

the logic being that the Wilson loops appearing in the Creutz ratio have average size $R$. In fact, taking the classical continuum limit, one finds that the Creutz ratio is a finite difference approximation:

$$
\hat{R}^{2} \tilde{\chi}(\hat{R}) \approx-\left.R T \frac{\partial^{2}}{\partial R \partial T} \ln W_{\text {cont. }}(R, T)\right|_{T=R},
$$

\footnotetext{
${ }^{1}$ In our analysis we take temporal extent $T=2 L$.
} 
where $W_{\text {cont. }}(R, T) \approx W(\hat{R}, \hat{T})$ is the Wilson loop in the continuum language. $T$ here should not be confused the temporal extent of the lattice.

In the Creutz ratio method for determining $g(L)$, we choose

$$
r=R / L=\text { fixed. }
$$

Note also that we have to deal with a fermion mass $m_{q}$, which we take to be the PCAC mass. Our approach will be to measure $g(L)$ at nonzero $m_{q}$ and then extrapolate to the $m_{q}=0$ limit.

The important thing that Bilgici et al. have noted is that at one loop in lattice perturbation theory, including the effect of bosonic zero modes,

$$
\hat{R}^{2} \tilde{\chi}_{1 \text {-loop }}(L, a \mid \hat{R})=k(r, N) g_{0}^{2}, \quad N=L / a
$$

which defines the quantity $k(r, N)$. Here, $g_{0}$ is the bare coupling. It was found in $[18,19]$ that in the limit of large $N, k(r, N) \rightarrow k(r)$, so that it is an $L$ and $a$ independent quantity in the continuum limit. Thus we write $k(r)$ in what follows, and will define the running coupling in terms of this $N \rightarrow \infty$ normalization factor.

Thus we can make an $L$-dependent nonperturbative definition of the running coupling $\bar{g}$ using the value of $\tilde{\chi}(R)$ obtained from a simulation with bare coupling $g_{0}:^{2}$

$$
\bar{g}^{2}(L) \equiv \lim _{a \rightarrow 0} \frac{1}{k(r)} \hat{R}^{2} \tilde{\chi}(L, a \mid \hat{R}), \quad \hat{R} a / L=\hat{R} / N=r=\text { fixed }
$$

To get a handle on $\bar{g}^{2}(L)$, we can study the scaling of the function:

$$
\left.g^{2}(N, r, L) \equiv \frac{1}{k(r)} \hat{R}^{2} \tilde{\chi}(N, a \mid \hat{R})\right|_{\hat{R}=r N}
$$

On the right-hand side, the nonperturbative $\tilde{\chi}(N, a \mid \hat{R})$ is evaluated with system size $L$. Clearly if $L$ is held fixed while $N$ is increased, the lattice spacing is decreasing toward the continuum limit. Thus $g^{2}(N, r, L)$ of (2.7) defines the running coupling in a particular (nonperturbative) scheme:

$$
\bar{g}^{2}(r \mid L) \equiv \lim _{N \rightarrow \infty} g^{2}(N, r, L) .
$$

The constant $r$ is part of the renormalization scheme.

A step-scaling analysis is used to follow the running of the coupling $\bar{g}$ with the scale $L$. We will not actually use step-scaling in order to search for the presence of an infrared fixed point. Rather, there is a simpler, qualitative behavior that we are looking for, as we now describe.

\subsection{Expectations}

In a confining theory, $g^{2}(x N, r, x L)>g^{2}(N, r, L)$ for $x>1$. This type of relationship means that if we hold $\beta$ fixed, the measured value of $g^{2}(N, r, L)$ increases as $N$ increases, since $L=N a$ will increase proportionately.

On the other hand, if there is an infrared fixed point, the ordering of curves with increasing $N$ reverses once the bare coupling $\beta$ goes past the fixed point $\left(\beta<\beta_{*}(N)\right)$, and $g^{2}(x N, r, x L)<$

\footnotetext{
${ }^{2}$ Often below we will use the lattice coupling $\beta=4 / g_{0}^{2}$.
} 
$g^{2}(N, r, L)$ occurs for $x>1$. Here we note that the fixed point $\beta_{*}$ depends on $N$, due to lattice artifacts. Indeed, such a "backwards running" was seen at strong couplings in the SU(3) theory with sixteen fundamental flavors in the work [21,20]. We expect something like this to also occur in the present theory at strong coupling, under the assumption that there is an infrared fixed point.

From the vantage point of the two behaviors that we have just presented, it is clear that a full blown step scaling analysis is not needed in order to detect the presence of an infrared fixed point. All that is needed is just to show the reversal of ordering with respect to $L$ at small $\beta$. This will be the focus of our analysis.

\section{Analysis}

\subsection{Smearing}

We follow Bilgici et al. and apply APE smearing $[24,25]$ for the links that are used in the calculation of Wilson loops. We take the smearing parameter to be $\alpha=0.5$. We find that two smearing steps works well for yielding the perturbatively required increase in $g^{2}(N, r, L)$ with $N$ at weak bare coupling. For this reason we use two smearing steps throughout this work.

\subsection{Interpolation}

In order to obtain a value of $g^{2}$ for $r=1 / 4$ with arbitrary $N$, it is necessary to interpolate to values of $\hat{R}$ that are not half-integral. For this purpose Bilgici et al. introduce a quadratic hypothesis. However, the obtained value is somewhat different if a cubic hypothesis is chosen. Furthermore, the result depends on which points are included in the fit. We have adopted the strategy of varying all these choices and then using the variation of $\mathrm{kg}^{2}$ as a measure of systematic error in the method. The mean value of $\mathrm{kg}^{2}$ is used as our final estimate.

\subsection{Results}

Now that we have outlined how we obtained the values of $k(r) g^{2}(N, r, L)$, we proceed to discuss our results. ${ }^{3}$ We have measured $\mathrm{kg}^{2}$ for lattices $N=10,12,16,20,24$ with bare coupling $\beta=2.25$. Note that this value of $\beta$ is at weaker coupling than the one where a bulk phase transition occurs, $\beta \approx 2$, as was found in [26]. It is also at a stronger coupling than the $\beta$ where there was some evidence for fixed point behavior in the Schrödinger functional study [15]. Thus this point is continuously connected to the continuum limit through finite mass, but is expected to be on the strong side of the infrared fixed point. That is, we have reason to suspect backwards running for the choice $\beta=2.25$. We have performed our simulations for five values of the bare mass $m_{0} a$, shown in Table 1. We have measured the PCAC mass $m_{q} a$ from the largest lattices $(N=24)$, where there is the least systematic error from finite volume. These are also given in Table 1. We then extrapolate $k(r) g^{2}(N, r, L)$ to the $m_{q}=0$ limit with quadratic and cubic fits to the data. Systematic errors are included in the uncertainties when performing the fit. In the case of the quadratic fit, we also consider the case where only the lightest four masses are included in the fit. The results for the measured values of $\mathrm{kg}^{2}$ are summarized in Table 2 and the results of the zero mass fits are

\footnotetext{
${ }^{3}$ We do not need the actual value of $k(r=1 / 4)$ since it is a fixed constant independent of $N$.
} 


\begin{tabular}{|c|c|}
\hline \hline$m_{0} a$ & $m_{q} a$ \\
\hline \hline-1.000 & $0.448406(5)$ \\
\hline-1.100 & $0.236337(4)$ \\
\hline-1.165 & $0.090917(2)$ \\
\hline-1.175 & $0.066873(6)$ \\
\hline-1.180 & $0.054890(3)$ \\
\hline \hline
\end{tabular}

Table 1: PCAC mass obtained from $24^{3} \times 48$ lattices.

\begin{tabular}{|c|c|c|c|c|c|}
\hline \hline & $m_{0} a=-1.000$ & $m_{0} a=-1.100$ & $m_{0} a=-1.165$ & $m_{0} a=-1.175$ & $m_{0} a=-1.180$ \\
\hline \hline $10^{3} \times 20$ & $0.6448(67)(0)$ & $0.4832(18)(0)$ & $0.38394(48)(0)$ & $0.36808(87)(0)$ & $0.3630(10)(0)$ \\
\hline $12^{3} \times 24$ & $0.7604(6)(32)$ & $0.5154(2)(32)$ & $0.3884(2)(16)$ & $0.37183(16)(66)$ & $0.35661(19)(35)$ \\
\hline $16^{3} \times 32$ & $0.9820(10)(37)$ & $0.6114(8)(31)$ & $0.3954(3)(18)$ & $0.3647(2)(13)$ & $0.35906(20)(97)$ \\
\hline $20^{3} \times 40$ & $1.2621(12)(21)$ & $0.6998(11)(28)$ & $0.4018(3)(13)$ & $0.3703(4)(22)$ & $0.3474(3)(17)$ \\
\hline $24^{3} \times 48$ & $1.6510(15)(7)$ & $0.8109(15)(12)$ & $0.4192(5)(10)$ & $0.3870(24)(7)$ & $0.3623(8)(18)$ \\
\hline \hline
\end{tabular}

Table 2: Results for $\mathrm{kg}^{2}$ for the various sizes of lattices and bare masses. The first error in $\mathrm{kg}^{2}$ is statistical, determined by jackknife analysis of fits to the Creutz ratios as described in the text. The second error in $\mathrm{kg}^{2}$ is systematic, obtained from varying the method of interpolation used to obtain the value at $\hat{R}=0.25(L / a)$.

presented in Table 3. The results of Table 2 are displayed along with the quadratic fits to all data in Fig. 1.

At large mass one sees the behavior characteristic of asymptotic freedom:

$$
g^{2}(24)>g^{2}(20)>g^{2}(16)>g^{2}(12)>g^{2}(10) .
$$

In the case of the quadratic fit to all data, when we extrapolate to the chiral limit the trend reverses:

$$
g^{2}(24) \approx g^{2}(20)<g^{2}(16)<g^{2}(12) \approx g^{2}(10) .
$$

Thus for this extrapolation we find backward running in the massless limit. However, in the other two extrapolations of Table 3, what we see is that there is no clear pattern, but instead a rough equality:

$$
g^{2}(24) \sim g^{2}(20) \sim g^{2}(16) \sim g^{2}(12) \sim g^{2}(10) .
$$

Thus what one has in this case is evidence for an absence of running.

The behavior that we have observed is consistent with the existence of an infrared fixed point. We regard our results as suggestive that Minimal Walking Technicolor does not actually walk, but is instead inside the conformal window. This is supportive of the findings of Schrödinger functional studies, but now by a different method.

\section{Conclusions}

We have found that the behavior of the coupling $g^{2}(N, r, L)$ is consistent with the existence of an infrared fixed point. Two refinements to the present study could be performed, which would 


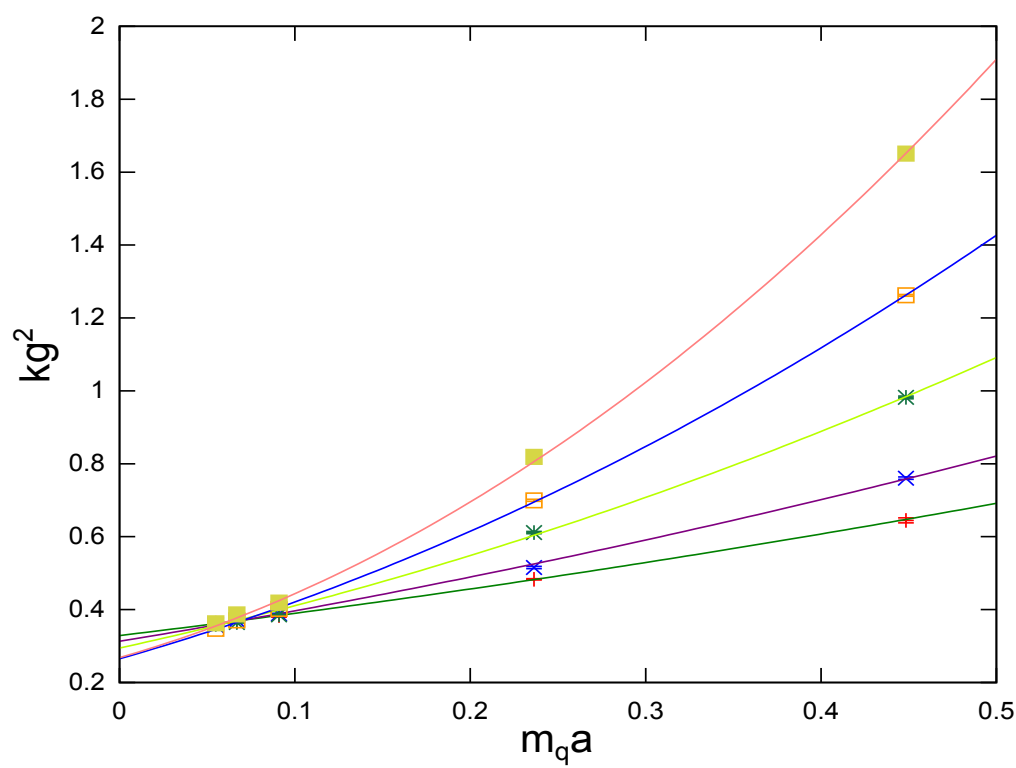

Figure 1: Values of $\mathrm{kg}^{2}$ for $\beta=2.25, r=1 / 4$. At large mass, $g^{2}$ increases with increasing $N$, so that the curves are ordered top to bottom, $N=24,20,16,12,10$. However, in the massless limit, the ordering of the curves shows a trend of reversal, indicating backward running.

\begin{tabular}{|c|c|c|c|c|c|c|}
\hline \hline & \multicolumn{2}{|c|}{ Quadratic, last 4 pts. } & \multicolumn{2}{c|}{ Quadratic, all 5 pts. } & \multicolumn{2}{c|}{ Cubic, all 5 pts. } \\
\hline & $\mathrm{kg}^{2}$ & $\chi^{2} /$ d.o.f. & $\mathrm{kg}^{2}$ & $\chi^{2} /$ d.o.f. & $\mathrm{kg}^{2}$ & $\chi^{2} /$ d.o.f. \\
\hline \hline $10^{3} \times 20$ & $0.3312(49)$ & 2.52 & $0.3288(26)$ & 1.75 & $0.3321(58)$ & 2.44 \\
\hline $12^{3} \times 24$ & $0.297(17)$ & 23.6 & $0.3130(95)$ & 25.7 & $0.291(20)$ & 21.3 \\
\hline $16^{3} \times 32$ & $0.319(20)$ & 12.7 & $0.295(11)$ & 19.0 & $0.327(23)$ & 11.4 \\
\hline $20^{3} \times 40$ & $0.284(15)$ & 5.04 & $0.2651(85)$ & 7.88 & $0.288(18)$ & 5.36 \\
\hline $24^{3} \times 48$ & $0.313(18)$ & 7.12 & $0.268(14)$ & 29.5 & $0.321(22)$ & 7.81 \\
\hline \hline
\end{tabular}

Table 3: Results for the zero mass extrapolation of $\mathrm{kg}^{2}$ for the various sizes of lattices.

hopefully yield firmer conclusions. First, smaller PCAC masses should be simulated so that a stronger case for what happens in the chiral limit can be made. Second, larger volumes such as $N=32$ should be simulated, so that the trends with respect to $N$ can be amplified.

\section{References}

[1] Leonard Susskind. Dynamics of Spontaneous Symmetry Breaking in the Weinberg- Salam Theory. Phys. Rev., D20:2619-2625, 1979.

[2] Steven Weinberg. Implications of Dynamical Symmetry Breaking: An Addendum. Phys. Rev., D19:1277-1280, 1979.

[3] Bob Holdom. Raising the Sideways Scale. Phys. Rev., D24:1441, 1981.

[4] Bob Holdom. Techniodor. Phys. Lett., B150:301, 1985. 
[5] Koichi Yamawaki, Masako Bando, and Ken-iti Matumoto. Scale Invariant Technicolor Model and a Technidilaton. Phys. Rev. Lett., 56:1335, 1986.

[6] M. Bando, K. Matumoto, and K. Yamawaki. Technidilaton. Phys. Lett., B178:308, 1986.

[7] Thomas W. Appelquist, Dimitra Karabali, and L. C. R. Wijewardhana. Chiral Hierarchies and the Flavor Changing Neutral Current Problem in Technicolor. Phys. Rev. Lett., 57:957, 1986.

[8] Thomas Appelquist and L. C. R. Wijewardhana. Chiral Hierarchies and Chiral Perturbations in Technicolor. Phys. Rev., D35:774, 1987.

[9] Thomas Appelquist and L. C. R. Wijewardhana. Chiral Hierarchies from Slowly Running Couplings in Technicolor Theories. Phys. Rev., D36:568, 1987.

[10] Estia Eichten and Kenneth D. Lane. Dynamical Breaking of Weak Interaction Symmetries. Phys. Lett., B90:125-130, 1980.

[11] Kenneth D. Lane and Estia Eichten. Two Scale Technicolor. Phys. Lett., B222:274, 1989.

[12] Francesco Sannino and Kimmo Tuominen. Techniorientifold. Phys. Rev., D71:051901, 2005.

[13] T. Appelquist, G. T. Fleming and E. Neil. Lattice Study of the Conformal Window in QCD-like Theories. Phys. Rev. Lett., 100:171607, 2008.

[14] Yigal Shamir, Benjamin Svetitsky, and Thomas DeGrand. Zero of the discrete beta function in SU(3) lattice gauge theory with color sextet fermions. Phys. Rev., D78:031502, 2008.

[15] Ari J. Hietanen, Kari Rummukainen, and Kimmo Tuominen. Evolution of the coupling constant in SU(2) lattice gauge theory with two adjoint fermions. Phys. Rev., D80:094504, 2009.

[16] Francis Bursa, Luigi Del Debbio, Liam Keegan, Claudio Pica, and Thomas Pickup. Running of the coupling and quark mass in SU(2) with two adjoint fermions. PoS, LAT2009:056, 2009.

[17] Thomas DeGrand, Yigal Shamir, and Benjamin Svetitsky. Infrared fixed point in SU(2) gauge theory with adjoint fermions. 2011.

[18] Erek Bilgici et al. A New Method of Calculating the Running Coupling Constant. PoS, LATTICE2008:247, 2008.

[19] Erek Bilgici et al. A new scheme for the running coupling constant in gauge theories using Wilson loops. Phys. Rev., D80:034507, 2009.

[20] Zoltan Fodor, Kieran Holland, Julius Kuti, Daniel Nogradi, and Chris Schroeder. Calculating the running coupling in strong electroweak models. PoS, LAT2009:058, 2009.

[21] Zoltan Fodor, Kieran Holland, Julius Kuti, Daniel Nogradi, and Chris Schroeder. Nearly conformal gauge theories in finite volume. Phys. Lett., B681:353-361, 2009.

[22] Hiroshi Ohki, Tatsumi Aoyama, Etsuko Itou, Masafumi Kurachi, C.-J.David Lin, et al. Study of the scaling properties in SU(2) gauge theory with eight flavors. PoS, LATTICE2010:066, 2010.

[23] Michael Creutz. Asymptotic Freedom Scales. Phys. Rev. Lett., 45:313, 1980.

[24] M. Albanese et al. Glueball Masses and String Tension in Lattice QCD. Phys. Lett., B192:163-169, 1987.

[25] M. Teper. An Improved Method for Lattice Glueball Calculations. Phys. Lett., B183:345, 1987.

[26] Simon Catterall, Joel Giedt, Francesco Sannino, and Joe Schneible. Phase diagram of SU(2) with 2 flavors of dynamical adjoint quarks. JHEP, 11:009, 2008. 